Einführung zum Thema

Onkologe $2020 \cdot 26: 391-392$

https://doi.org/10.1007/s00761-020-00751-4

(C) Springer Medizin Verlag GmbH, ein Teil von Springer Nature 2020

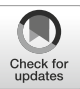

\author{
S. Sahm ${ }^{1,2} \cdot$ B. van Oorschot ${ }^{3} \cdot$ E. C. Winkler $\cdot$ J. Schildmann ${ }^{5} \cdot$ K. Höffken ${ }^{6}$ \\ 'Senckenbergisches Institut für Geschichte und Ethik der Medizin, Goethe-Universität, Frankfurt, \\ Deutschland \\ ${ }^{2}$ Medizinische Klinik I, Ketteler Krankenhaus, Offenbach, Deutschland \\ ${ }^{3}$ Universitätsklinikum Würzburg, Würzburg, Deutschland \\ ${ }^{4}$ Ruprecht-Karls-Universität Heidelberg, Heidelberg, Deutschland \\ ${ }^{5}$ Universitätsmedizin Halle, Halle, Deutschland \\ ${ }^{6}$ Berlin, Deutschland
}

\title{
Nicht alles, was machbar ist, ist auch vertretbar
}

\section{Ethische Herausforderungen in der Onkologie}

Medizinisches Handeln versteht sich nicht von selbst. Andernfalls wären ärztliche Gelöbnisse überflüssig. Seit den Zeiten des Hippokrates ist bekannt, wie sehr die Ausrichtung medizinischen Handelns einer ethischen Grundlage und einer Rechtfertigung bedarf. Das zeigt sich besonders dann, wenn ethische Überzeugung gebietet, spezifische Handlungen zu unterlassen.

Ethik geht immer dem Recht voraus. Die Einsicht ist Fundament einer freien und liberalen Gesellschaft [1]. Daher müssen ethische Überlegungen der Entscheidung über Richtlinien und ggf. gesetzliche Regelungen vorangestellt werden.

Ein Blick in den Alltag der onkologischen Medizin in Kliniken, Forschungseinrichtungen und Praxen entdeckt vielfältige ethische Probleme. Es ist Tradition der Medizin, sich auch diesen Fragen zu stellen.

Die Beiträge des vorliegenden Hefts wurden von anerkannten Autoren auf dem Gebiet der medizinischen Ethik verfasst. Gleichwohl kann nicht der Anspruch erhoben werden, alle ethischen Konflikte, die in der onkologischen Praxis begegnen, in einer Ausgabe zu diskutieren. Doch sollen aktuelle Fragen umfassend reflektiert und dargestellt werden. Die Auseinandersetzung mit Argumenten im Für und Wider ist Voraussetzung, ethisch fundierte Entscheidungen zu treffen.

Die Autoren um Christin Kohrs befassen sich mit den klinisch-ethischen Aspekten der Komplementärmedizin. Der Begriff ist nicht scharf definiert, was erhebliche Schwierigkeiten auch für die ethische Bewertung der Komplementärmedizin nach sich zieht. Nicht wenige Patienten knüpfen an solche Behandlung große Erwartung. Fast jeder Betroffene wünscht, darüber mit Ärztinnen und Ärzten zu sprechen. Ein solches Gespräch kann Ausgangspunkt für eine Kommunikation mit den Patienten werden, die häufig weit über konkrete Verfahren der Komplementärmedizin hinausgeht.

Bernd Alt-Epping beschreibt die Wege der Entscheidungsfindung in der palliativen Onkologie. Wo liegen die Grenzen der Therapie angesichts vielfältiger Belastungen, die mit ihr einhergehen? Kein Flow-Sheet aus dem Lehrbuch hilft weiter. Bedeutsam ist es, die Perspektive der Patienten als essenziellen Bestandteil in die Überlegungen einzubeziehen. Dies verlangt nicht allein das Recht. Alle Maßnahmen sind nur dann medizinisch und ethisch gerechtfertigt, d.h. indiziert, wenn sie den Betroffenen im Einzelfall auch dienlich sein können. Ohne Zweifel stehen hinter diesen Überlegungen immer auch normative Einschätzungen. Diese gilt es zu begründen und in der Kommunikation mit den Betroffenen zum Vorschein zu bringen.

Noch immer hegen viele Patienten das Vorurteil, die Medizin kenne keine Grenzen. Doch mittlerweile darf vorausgesetzt werden: Begrenzungen medizinischer Maßnahmen gehören zur ärztlichen Routine. Dennoch ist es für viele Menschen wichtig, ihre Wünsche im Blick auf die Behandlung am Lebensende in Vorabverfügungen für den Fall festzulegen, wenn sie vielleicht nicht mehr selbst entscheiden können. Der Umgang mit Patientenverfügungen und Vorsorgevollmachten bedarf nicht nur der Kenntnisse über deren rechtliche Wirksamkeit. Vielmehr sind Einfühlungsvermögen und Offenheit vonnöten, die Wünsche der Betroffenen zu hören. Nicht zuletzt kann das Gespräch über eine Patientenverfügung als Instrument genutzt werden, die Kommunikation mit dem Patienten zu vertiefen. Dies stellen Tanja Henking und Birgitt van Oorshot in ihrer Arbeit dar.

Katja Mehlis, Bastian Surmann und Eva Winkler befassen sich mit einem bislang vernachlässigten Problem den finanziellen Auswirkungen einer Krebserkrankung für die Betroffenen. Hintergrund ist eine empirische Untersuchung am Nationalen Centrum für Tumorerkrankungen in Heidelberg. Finanzielle Nöte durch Verlust der Arbeitsfähigkeit betreffen nicht nur Patienten in 
den USA, wo die sozialen Sicherungssysteme weit weniger dicht geknüpft sind. Die Autoren diskutieren die sozialethischen Implikationen, sofern sich dieser Trend für ganz Deutschland nachweisen lässt.

Wesentlicher Treiber des Fortschritts in der Onkologie ist die genombasierte Präzisionsmedizin. Sie verspricht vielen Patienten eine auf sie zugeschnittene Therapie. Voraussetzung ist die fundierte Analyse und Anwendung der Erkenntnisse, wie es in molekularen Tumorboards geschieht. Doch ergeben sich neue ethische Herausforderungen, wie Christoph Schickhardt, Peter Horak, Stefan Fröhling und Eva Winkler darstellen. Klärung der Verantwortlichkeiten, der Umgang mit akzidentiell entdeckten $\mathrm{Zu}$ satzbefunden, Schweigepflicht und die verantwortliche Weitergabe von Daten müssen in geeigneter Weise adressiert und bedacht werden.

Schließlich gilt es, die aktuellen Kontroversen um das Maß medizinischer Behandlung am Lebensende zu betrachten. Nach Maßgabe der Verlautbarung ärztlicher Organisationen ist der freiwillige Verzicht auf Nahrung und Flüssigkeit (FVNF) eine vom Suizid unterschiedene Handlung. Doch stellt sie insbesondere für Pflegende eine besondere Herausforderung und Belastung dar. Sie sind nahe am Patienten dran. In den letzten Jahren wurden Grundlagen der Pflegeethik herausgearbeitet. Wie passen FVNF und die Sorge um die Patienten zusammen? $\mathrm{Ka}$ rola Selge und Margit Haas analysieren den FVNF im Kontext der etablierten Pflegeethik. Es ist wichtig zu wissen, wie die Pflegenden über die Entscheidung zur Begleitung eines FVNF denken und empfinden. Die Autorinnen stellen klar, dass Begleitung von Patientinnen und Patienten, die sich angesichts einer fortgeschrittenen terminalen Erkrankung zum FVNF entschlossen haben, mit den etablierten Prinzipien der Pflegeethik in Einklang steht.

Und zuletzt bedarf der auch in der Öffentlichkeit viel beachtete Streit um die ärztliche Assistenz beim Suizid (international: "physician-assisted suicide“ [PAS]) einer eingehenden Betrachtung. Stephan Sahm analysiert die gesellschaftliche Auseinandersetzung darüber im
Lichte medizinischer Ethik. Erst unlängst hat das Bundesverfassungsgericht das gesetzliche Verbot geschäftsmäßiger Suizidhilfe aufgehoben. Das befreit Ärztinnen und Ärzte nicht von der Aufgabe, zur Frage der Beihilfe zum Suizid Stellung zu nehmen. Onkologisch tätige Ärztinnen und Ärzte sehen sich immer wieder mit suizidalem Begehren konfrontiert. Wie ist damit umzugehen? Was sind die Gründe für die Ablehnung der ärztlichen Suizidhilfe durch die verfasste Ärzteschaft und den Weltärztebund? Ungeachtet der Entscheidungen des Verfassungsgerichts und der Vielfalt ethischer Überzeugungen in der Gesellschaft ist hier die Ärzteschaft gefordert. Die Rückweisung ärztlicher Suizidassistenz ist ein Akt der Gefahrenabwehr, denn die Auswertung empirischer Untersuchen begründet die Annahme, dass das Angebot die Rate der Suizide steigen lässt. Zudem widerspricht sie etablierten Prinzipien medizinischer Ethik. Und weiter: Es gibt keine medizinische Notwendigkeit, suizidalem Begehren Folge $\mathrm{zu}$ leisten, wenn umfassende palliativmedizinische Betreuung gewährleistet ist.

Bei allen aufgegriffenen Themen wird zum einen deutlich, dass jede medizinische Entscheidung eine normative Komponente hat, die es gilt explizit zu machen, zu begründen und mit den Patienten zu kommunizieren. Zum anderen, dass der erwünschte kurze Weg zwischen Forschung und Versorgung eigene ethische Fragen aufwirft und dass eine Reihe ethischer Fragen, wie etwa die nach der finanziellen Absicherung bei Krankheit oder der ärztlichen Beihilfe zum Suizid, im gesellschaftlichen Diskurs beantwortet werden muss.

S. Sahm, B. van Oorschot, E.C. Winkler, J. Schildmann (Schriftleiter)

K. Höffken (für die Herausgeber)

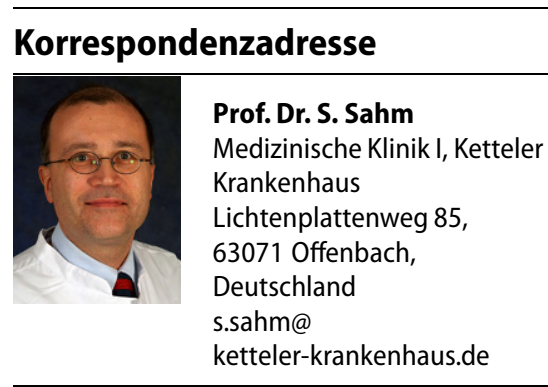

Interessenkonflikt. S. Sahm, B. van Oorschot, E.C. Winkler, J. Schildmann und K. Höffken geben an, dass kein Interessenkonflikt besteht.

\section{Literatur}

1. Kirchhof P (2005) Das Recht auf Leben und Gesundheit für alle Generationen. Z Med Ethik 51:229-241 